\section{Low-Rhes approach to Huntington's}

\section{By Tim Fulmer, Senior Writer}

A central mystery of Huntington's disease has been why the mutant form of the huntingtin protein responsible for the condition is found throughout the body but only causes pathology in the brain's corpus striatum. Researchers at The Johns Hopkins University School of Medicine may have the answer-a protein called Rhes that is specific to the corpus striatum and mediates the neurotoxic effects of mutant huntingtin protein. ${ }^{1}$

The target looks to be druggable, thus potentially expanding the current target landscape in HD (see Table 1, "Huntington's disease pipeline"), and could be explored using adaptations to existing animal models.

The group searched for proteins or factors that interact with mutant huntingtin (HTT) in the striatum and might at least partly explain the striatal-specific neurotoxicity of the mutant. They eventually zeroed in on Rhes (RASD2), a Ras-related guanosine triphosphate-binding protein known for its enrichment in the striatum but otherwise not previously implicated in HD pathogenesis.

Studies in cell culture confirmed that Rhes bound mutant HTT. In vitro, cells expressing Rhes in the presence of mutant HTT had significantly lower survival than cells that only had mutant HTT $(p<0.005)$.

To get a better handle on the mechanism of neurotoxicity, the Hopkins group next looked at the influence of Rhes on mutant HTT aggregation. Aggregates in the striatum are a hallmark of HD, although their causal role in pathology is up for debate. Competing models of $\mathrm{HD}$ describe aggregation as pathogenic, beneficial or merely incidental to disease. ${ }^{2}$

The Hopkins researchers chose to express Rhes and mutant HTT in human embryonic kidney cells. Those cells normally don't express the two proteins and therefore have no interfering background activity.

The results point away from the pathogenic roles of aggregates.

Rhes expression led to significantly higher soluble mutant HTT and significantly lower aggregated mutant HTT than occurred in the absence of Rhes ( $p<0.005$ for both). Soluble mutant HTT showed increased levels of sumoylation, a post-translational process in which a small ubiquitin-like modifier (SUMO) is added to multiple lysine residues.

In vitro, cells with blocked sumoylation of mutant HTT or deactivated Rhes had significantly lower levels of soluble mutant HTT and greater cell survival than wild-type cells $(p<0.005)$.

Taken together, the findings suggest a cascade of events whereby
Rhes binds to mutant HTT and promotes its sumoylation at three different lysine residues. Those modifications then trigger the formation of soluble mutant HTT instead of the aggregated form, leading to neurotoxicity and cell death.

The findings were published in Science.

"The Science paper is potentially a major advance in the field because, for the first time, it implicates a single protein, Rhes, in the selective vulnerability of the striatum to mutant huntingtin," said Robert Friedlander, professor of neurosurgery at the Harvard Medical School and director of the Neuroapoptosis Laboratory at Brigham and Women's Hospital. "Moreover, the paper provides initial evidence that Rhes is a druggable target."

\section{Allowing for aggregates}

The results suggest that targeting the cascade early could prevent build-up of soluble mutant HTT and be neuroprotective. The findings also suggest that soluble mutant HTT is a better target than aggregates of the protein. In addition, data in the Science article add to a growing body of research showing that aggregates and inclusion bodies are not necessarily a bad thing in HD.

"The results are consistent with previous findings that inclusion body formation may serve as a protective response by neurons against more soluble and toxic forms of mutant huntingtin," said Steven Finkbeiner of the University of California, San Francisco.

Previous microscopy studies by Finkbeiner and colleagues showed that cultured rat striatal neurons that produced HTT inclusion bodies survived significantly longer than adjacent neurons that lacked inclusion bodies $(p<0.0003) .^{3}$

Finkbeiner is senior investigator and associate director at the Gladstone Institute of Neurological Disease, director of the Taube-Koret Center for Huntington's Disease Research and professor of neurology and physiology at UCSF.

According to J. Lawrence Marsh, the Science paper highlights the contribution of post-translational modifications such as sumoylation to the toxicity and potential pathogenicity of the huntingtin protein. "If inhibiting Rhes and/or sumoylation can slow the formation of toxic forms of huntingtin, then this and other studies implicating sumoylation in pathogenesis will have opened up a new therapeutic opportunity for ameliorating Huntington's disease," he said.

Marsh and colleagues previously reported that sumoylation of an HTT fragment enhanced neurodegeneration in a Drosophila model of HD. ${ }^{4}$ Marsh is professor of developmental and cell biology and director of the University of California, Irvine's Developmental Biology Center.

Srinivasa Subramaniam, lead author on the Science paper and postdoctoral researcher in Solomon Snyder's lab, told SciBX the group's first priority is now to design an HD mouse model to further study Rhes.

The good news is that Rhes knockout mice already exist, ${ }^{5}$ so a clear next step would be crossing the knockouts with HD models, he said.

"We could then compare the gross pathology and shrinkage of the 
Table 1. Huntington's disease pipeline. At least 14 compounds are in development for Huntington's disease (HD).

\begin{tabular}{|c|c|c|c|}
\hline Company & Product & Description & Status \\
\hline Amarin Corp. plc (NASDAQ:AMRN) & AMR101 & Eicosapentaenoic acid derivative & Under review in EU \\
\hline NeuroSearch A/S (CSE:NEUR) & Pridopidine (ACR16) & Dopamine stabilizer & Phase III \\
\hline Avicena Group Inc. (Pink:AVCE) & HD-02 & Therapeutic that incorporates a clinical form of creatine & Start Phase III by $4 \mathrm{Q} 09$ \\
\hline $\begin{array}{l}\text { Medivation Inc. (NASDAQ:MDVN)/ } \\
\text { Pfizer Inc. (NYSE:PFE) }\end{array}$ & Dimebon & Blocks an undisclosed target involving mitochondrial pores & Phase II \\
\hline $\begin{array}{l}\text { Prana Biotechnology Ltd. } \\
\text { (ASX:PBT; NASDAQ:PRAN) }\end{array}$ & PBT2 & Metal-protein-attenuating compound (MPAC) & Phase I \\
\hline $\begin{array}{l}\text { Neuera Pharmaceuticals Inc., a subsidiary of } \\
\text { Accera Inc. }\end{array}$ & AC-0523 & Compound that addresses mitochondrial dysfunction in HD & Preclinical \\
\hline Adeona Pharmaceuticals Inc. (NYSE-A:AEN) & Oral TTM & Oral tetrathiomolybdate anti-copper agent & Preclinical $^{\mathrm{A}}$ \\
\hline $\begin{array}{l}\text { Alnylam Pharmaceuticals Inc. (NASDAQ: } \\
\text { ALNY)/Medtronic Inc. (NYSE:MDT) }\end{array}$ & ALN-HTT & Small interfering RNA against the huntingtin (HTT) gene & Preclinical \\
\hline Ceregene Inc. & CERE-120 & $\begin{array}{l}\text { Adeno-associated virus (AAV) type } 2 \text { vector encoding } \\
\text { neurturin }(N T N) \text { gene }\end{array}$ & Preclinical \\
\hline Cortex Pharmaceuticals Inc. (NYSE-A:COR) & CX929 & $\begin{array}{l}\text { An ampakine that is a positive modulator of AMPA } \\
\text { glutamate receptors }\end{array}$ & Preclinical \\
\hline Enkam Pharmaceuticals A/S & Defakin 1 & Fibroblast growth factor receptor activator & Preclinical \\
\hline Neurologix Inc. (OTCBB:NRGX) & dXIAP & Mutated form of the gene $\mathrm{C} 15$ delivered by AAV vector & Preclinical \\
\hline Repligen Corp. (NASDAQ:RGEN) & $\begin{array}{l}\text { Histone deacetylase } \\
\text { inhibitor } 4 \mathrm{~b}\end{array}$ & Benzamide-type histone deacetylase (HDAC) inhibitor $4 \mathrm{~b}$ & Preclinical \\
\hline Targeted Genetics Corp. (NASDAQ:TGEN) & siRNA/AAV vector & AAV vector delivering siRNA targeting $H T T$ & Preclinical \\
\hline
\end{tabular}

An a recent $10-\mathrm{K}$ filing, Adeona said it is seeking partners for further development.

striatum in Rhes-deficient HD models with models expressing normal levels of Rhes," Subramaniam said. "That would potentially provide additional genetic evidence in support of pharmacologically suppressing Rhes activity in neurodegenerative disease."

RNAi experiments also could provide support for targeting Rhes in HD, said Maria Pennuto, group leader in the Department of Neuroscience at the Italian Institute of Technology.

In the Science article, the Hopkins researchers reported that depleting Rhes levels with small hairpin RNA improved survival of cultured neuronal cells compared with control shRNA treatment.

"It now remains to be determined whether shRNA knockdown of Rhes would be effective in animal models of Huntington's disease," said Pennuto.

\section{Repressing Rhes}

In parallel with the genetics studies in animals, Subramaniam and colleagues hope to identify small molecules that could target Rhes or other proteins with which it interacts.

In the latter category, a prime target could be ubiquitin-conjugating enzyme E2I (UBE2I; UBC9), a key protein in the sumoylation pathway that is bound by Rhes. Blocking Rhes-UBC9 interactions in the striatum could potentially reduce sumoylation of mutant HTT and lead to lower levels of the neurotoxic soluble form of the protein, said Subramaniam.

He acknowledged that targeting protein-protein interactions is a daunt- ing task and said it might be better to go upstream and directly inactivate Rhes before it interacts with mutant HTT or the sumoylation pathway.

Johns Hopkins owns patents covering the Rhes-targeting strategy in neurodegenerative diseases, and those patents are available for licensing, according to Subramaniam.

Fulmer, T. SciBX 2(25); doi:10.1038/scibx.2009.993

Published online June 25, 2009

\section{REFERENCES}

1. Subramaniam, S. et al. Science; published online June 5, 2009; doi:10.1126/science. 1172871

Contact: Solomon Snyder, The Johns Hopkins University

School of Medicine, Baltimore, Md.

e-mail: ssnyder@jhmi.edu

2. Orr, H. Nature 431, 747-748 (2004)

3. Arrasate, M. et al. Nature 431, 805-810 (2004)

4. Steffan, J. et al. Science 304, 100-104 (2004)

5. Spano, D. et al. Mol. Cell Biol. 24, 5788-5796 (2004)

\section{COMPANIES AND INSTITUTIONS MENTIONED}

Brigham and Women's Hospital, Boston, Mass.

Gladstone Institute of Neurological Disease, San Francisco, Calif.

Harvard Medical School, Boston, Mass.

Italian Institute of Technology, Genoa, Italy

The Johns Hopkins University School of Medicine, Baltimore, Md. Taube-Koret Center for Huntington's Disease Research, San Diego, Calif.

University of California, Irvine, Calif.

University of California, San Francisco, Calif. 